\title{
Emulsifying conditions and processing parameters optimisation of kenaf seed oil-in- water nanoemulsions stabilised by ternary emulsifier mixtures
}

\begin{abstract}
Kenaf (Hibiscus cannabinus L.) seed oil has been proven for its multi-pharmacological benefits; however, its poor water solubility and stability have limited its industrial applications. This study was aimed to further improve the stability of pre-developed kenaf seed oil-in-water nanoemulsions by using food-grade ternary emulsifiers. The effects of emulsifier concentration $(1,5,10,15 \% \mathrm{w} / \mathrm{w})$, homogenisation pressure $(16,000,22,000$, $28,000 \mathrm{psi}$ ), and homogenisation cycles (three, four, five cycles) were studied to produce high stability of kenaf seed oil-in-water nanoemulsions using high pressure homogeniser. Generally, results showed that the emulsifier concentration and homogenisation conditions had great effect $(p<0.05)$ on the particle sizes, polydispersity index and hence the physical stability of nanoemulsions. Homogenisation parameters at $28,000 \mathrm{psi}$ for three cycles produced the most stable homogeneous nanoemulsions that were below $130 \mathrm{~nm}$, below 0.16 , and above $-40 \mathrm{mV}$ of particle size, polydispersity index, and zeta potential, respectively. Field emission scanning electron microscopy micrograph showed that the optimised nanoemulsions had a good distribution within nano-range. The optimised nanoemulsions were proved to be physically stable for up to six weeks of storage at room temperature. The results from this study also provided valuable information in producing stable kenaf seed oil nanoemulsions for the future application in food and nutraceutical industries.
\end{abstract}

Keyword: Kenaf (Hibiscus cannabinus L.) seed oil; Homogenisation parameters; Nanoemulsions; Stability; Field emission scanning electron microscopy 